\title{
Spontaneous Multivessel Dissection in Young Woman - What is the Best Treatment Strategy?
}

Trendafilova D, Jorgova J, Nachev G

Clinic of Cardiology and Cardiosurgery, University Hospital " St Ekatherina" Bulgaria

\begin{abstract}
This report describes the case of a 44 year-old female who was referred to our hospital after cardiac arrest and successful resuscitation. Coronary angiography revealed spontaneous dissection in a large segment of LM to LAD. The patient was referred to emergent coronary artery by-pass surgery. Four years later she was admitted with clinical and ECG findings for STEMI. Coronary angiography showed patent left coronary arteries, occluded venous graft to Rcx, occluded LIMA-LAD and spontaneous proximal dissection and occlusive thrombus in RCA. The vessel was successfully re-canalized and stented. Two years later the patient was admitted for coronary angio with symptoms of progressive heart failure. The diagnostic angio showed patent coronary arteries, occluded grafts and left ventricle aneurysm with EF $38 \%$ and severe MR. After discussion with the surgeons she received a mitral valve reconstruction.
\end{abstract}

There are no specific guidelines how to manage these patients. For cases of multivessel disease and particularly when the left main or LADis affected, cardiac surgery/or PCI/ may be the therapy of choice. There are reported cases where treatment of LM dissection has been conducted successfully and conservatively.

Keywords: Spontaneous Coronary Artery Dissection (SCAD); Coronary Artery By-pass surgery (CABG); Percutaneous Coronary Intervention $(\mathrm{PCI})$

\section{Acronyms}

ACS: Acute Coronary Syndrome; BMS: Baremetal Stent; CABG: Coronary Artery By-pass Graft; CHF: Chronic Heart Failure; DAPT: Dual Antiplatelet Therapy; ECG: Electrocardiography; EF: Ejection Fraction; LAD: Left Descending Artery; LCA: Left Coronary Artery; LIMA: Left Internal Mammary Artery; LM: Left Main; LV: Left Ventrical; MR: Mitral Regurgitation; PCI: Percutaneous coronary intervention; RCA: Right Coronary Artery; Rcx: Circumflex Artery; SCAD: Spontaneous Coronary Artery Dissection; STEMI: ST segment elevation myocardial infarction; SVG: Saphenous Venous Graft

\section{Introduction}

Spontaneous Coronary Artery Dissection (SCAD) is a rare but important cause of acute coronary syndromes. SCAD can cause unstable angina, acute myocardial infarction and sudden death. Predisposing factors include sex, atherosclerosis, the peripartum period and structural and inflammatory conditions affecting the arterial wall. The disease affects women more often than men. Spontaneous coronary artery dissection was found to occur most often in the first 3 weeks after giving birth. Genetic diseases that cause problems with the body's connective tissues such as Marfan syndrome, very high blood pressure in progressive arteriosclerotic changes, extreme physical exertion as well as cocaine use are other risk factors prompting spontaneous coronary artery dissection. The diagnosis of coronary dissection is usually made by coronary angiography. Therapeutic options include medical therapy, percutaneous coronary intervention and surgery. We present a case of a young woman with recurrent spontaneous coronary artery dissection in different territories.

\section{Definition}

Coronary artery dissection can occur spontaneously or as a consequence of chest trauma, cardiac surgery, coronary angiography, coronary intervention or as extension of aortic dissection. Dissection of the coronary artery results in separation of the layers of the arterial wall creating a false lumen. Hemorrhage into the false lumen can impinge upon the true lumen of the coronary artery impairing blood flow and causing myocardial ischemia, infarction, or sudden death [1].

\section{Case}

A 44 year female was transferred to our hospital with ACS after cardiac arrest and successful resuscitation. She had no history of any known risk factors such as arterial hypertension, dyslipidemia, familial disease or any comorbidities. She had no hormonal disorders and had not used contraceptives. No genetic abnormalities or signs for chronic infection could be found.

Lab results showed normal enzyme markers. ECG showed negative T-waves in I; AVL; V1-V3.

ECHO showed septoapical dyskinesia EF-64\%; MR (mitral regurgitation)-II degree.

Coronary angiography showed large dissection involving left main and proximal and mid segment LAD (Figure 1) with proximal occlusion of RCX (Figure 2) and normal RCA (Figure 3). An AV fistula originating from RD1 to LA was also found.

The patient was referred for urgent CABG (Coronary Artery

*Corresponding author: Diana Trendafilova, Clinic of Cardiology and Cardiosurgery, University Hospital "St Ekatherina", 52 ABul. Pencho Slavejkov 1431 Sofia, Bulgaria, Tel: +359 888275738; Fax: +35929549057; E-mail: dianalaztr@yahoo.com

Received February 27, 2014; Accepted April 19, 2014; Published April 19, 2014

Citation: Trendafilova D, Jorgova J, Nachev G (2014) Spontaneous Multivessel Dissection in Young Woman - What is the Best Treatment Strategy? J Vasc Med Surg 2: 132. doi: 10.4172/2329-6925.1000132

Copyright: (C) 2014 Trendafilova D, et al. This is an open-access article distributed under the terms of the Creative Commons Attribution License, which permits unrestricted use, distribution, and reproduction in any medium, provided the original author and source are credited. 
Citation: Trendafilova D, Jorgova J, Nachev G (2014) Spontaneous Multivessel Dissection in Young Woman - What is the Best Treatment Strategy? J Vasc Med Surg 2: 132. doi: 10.4172/2329-6925.1000132

Page 2 of 3

Bypass Graft). She received LIMA (Left internal mammary artery graft) to LAD and SVG (saphenous venous graft) to RCX. She was discharged on the 8th postoperative day with uncomplicated post-operative period and $\mathrm{EF} 50 \%$.

Four years later she was again transferred from a peripheral hospital with STEMI in inferior wall. Coronary angiography showed patent native LCA with small residual non-occlusive dissection in LM (Figure 4) occluded venous graft to Rcx, occluded LIMA-LAD (Figure 5 ) and a large spontaneous dissection and occlusive thrombus in RCA (Figure 6).

After several thrombaspirations and application of full dose IIb/IIIa inhibitors, the vessel was successfully re-canalized and the proximal segment was stented with a BMS 3,5/24 stent and an end result with TIMI II (Figure 7). Left ventrical angiography showed EF-40\% with infero-basal hypo kinesis and MR-up to II degree (Figures 8a and 8b).

During the next days we had a complete resolution of ECG and enzyme markers.

She was discharged with persistent LV dysfunction and EF 40\%, MR II and therapy of DAPT; Beta- blocker; ACE inhibitor and high dose statin was recommended.

Two years later the patient was angina free but with symptoms of progressive congestive heart failure and a MR III.

Subsequent coronary angiography - both LCA and RCA, were patent with no in-stent restenosis in the RCA stent. MR III was found.

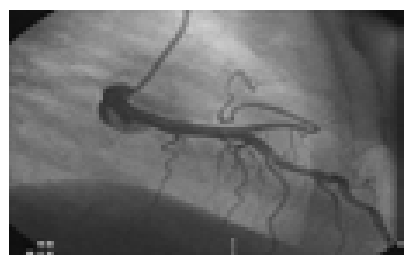

Figure 1: Large dissection involving left main and proximal and mid segment LAD.

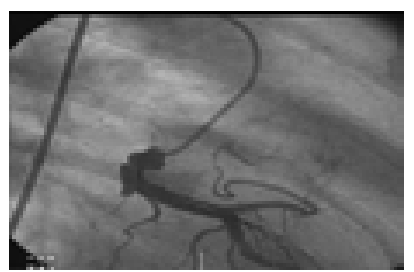

Figure 2: Proximal occlusion of RCX.

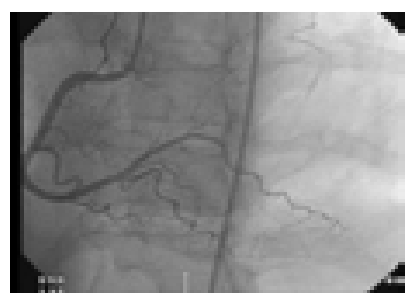

Figure 3: Normal RCA.

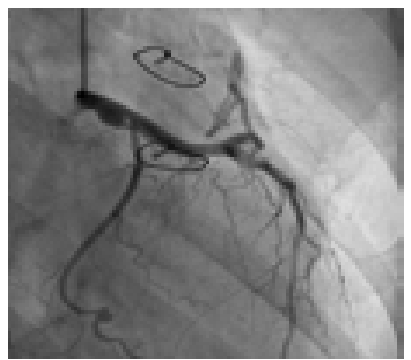

Figure 4: LCA with small residual non-occlusive dissection in LM.

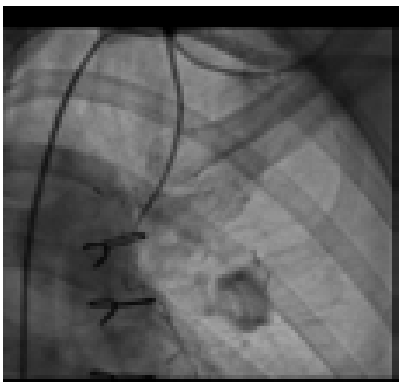

Figure 5: Ocluded LIMA-LAD.

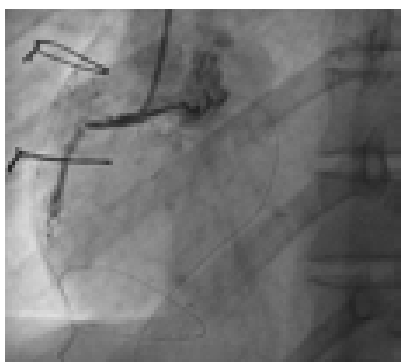

Figure 6: Large spontaneous dissection and occlusive thrombus in RCA

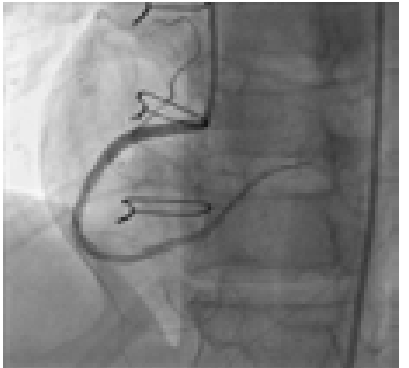

Figure 7: RCA after BMS final result.

After discussion with the surgeons she received a mitral valve reconstruction.

Follow-up one year later she remains angina free and symptoms of CHF, NYHA - II, EF - $45 \%$ with no MR.

\section{Discussion}

Spontaneous coronary artery dissection (SCAD) remains a rare, 


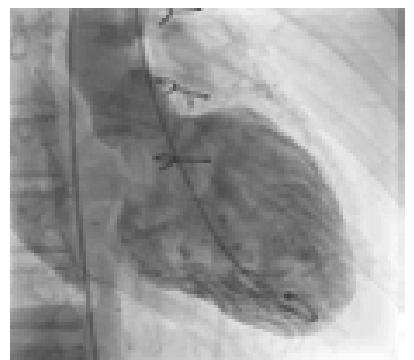

Figure 8a: Left ventrical angiography.

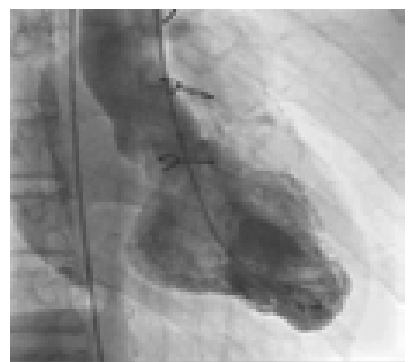

Figure 8b: Left ventrical angiography.

but challenging entity. In 1931, Pretty [2] published the first case and Forker et al. [3] reported the first angiographic diagnosis. Since then less than 400 cases have been reported. Previous publications on SCAD are based on single cases or relatively small series with retrospective design and without long-term follow-up.

There are three main subgroups of patients who present with coronary artery dissection [4]. The first group of patients includes those with underlying atherosclerosis; it accounts for about $45 \%$ of cases. The second group includes patients whose arteries dissect in association with pregnancy or the postpartum state, which accounts for $12 \%$ of cases. The third group includes those without any identifiable risk factors and accounts for $34 \%$ of the cases. Our patient falls into this 'idiopathic' group. Our patient's case is also interesting as a documentation of significant recurrent disease. She had two separate events. The incidence of recurrent SCAD is unknown.

The 'idiopathic' patient group probably represents a group of different connective tissue disorders or vasculitis presenting with a common clinical syndrome.

The overall prognosis of SCAD is unknown because the groups and literature reviews are heterogenous and treatment options vary.

There are no randomized trials of coronary dissection treatment. The literature consists of case reports and case series. Treatment options for SCAD include medical therapy, percutaneous coronary intervention (PCI) or coronary artery bypass graft surgery. The decision to treat medically or perform percutaneous intervention or surgery must be individualized based on both clinical and angiographic factors. Dissection of large coronary arteries causing persistent ischemia is usually treated with percutaneous intervention or surgery, while dissections of small vessels are treated medically.

When there is no evidence of persistent ischemia or hemodynamic instability, medical therapy alone may be utilized. Treatment of spontaneous coronary dissection is similar to treatment of acute coronary syndromes. This includes anticoagulation with heparin or enoxaparin, aspirin, clopidogrel, beta-blockers and nitrates. Calcium channel blockers may be used to treat spasm. Widespread use of coronary angiography in ACS has increased the number of diagnosed cases.

Our decision to send the patient for surgery was based on the length of the dissection which included the left main, proximal and mid segment of the LAD and the occlusion of the RCX. Early occlusion of the LIMA and SVG grafts though could be explained with the healing of the vessel as a result of the natural evolution of the disease and the creation of competitive flow. This leads to the question - could we have risked waiting and tried more aggressive conservative therapy?

Another interesting question to which we don't have an answer is the reason for the recurrence of the process in a different vascular territory. Such cases are very rare in the literature.

\section{Summary}

Management of patients with SCAD remains highly controversial.

All of the therapies were reported to be successful in case reports and case series. However, more substantial evidence from controlled studies is lacking. The literature on SCAD is growing; however, there is still lack of understanding regarding the pathophysiology, prognosis and optimal treatment options for this disease. In most of the cases for this disease at least one of the risk factors known to the literature is present.

Our case report of a young woman with SCAD is different as none of the known risk factors are found to explain the disease. It is also interesting for a number of reasons - including the pattern of artery involvement and decision for management options.

\section{References}

1. Butler R, Webster MW, Davies G, Kerr A, Bass N, et al. (2005) Spontaneous dissection of native coronary arteries. Heart 91: 223-224.

2. Pretty H (1931) Dissecting aneurysms of coronary artery in woman aged. BMJ 42: 667.

3. Forker AD, Rosenlof RC, Weaver WF, Carveth SW, Reese HE (1973) Primary dissecting aneurysm of the right coronary artery with survival. Chest 64:656658.

4. Hering D, Piper C, Hohmann C, Schultheiss HP, Horstkotte D (1998) [Prospective study of the incidence, pathogenesis and therapy of spontaneous by coronary angiography diagnosed coronary artery dissection]. Z Kardiol 87 961-970. 